\title{
Rotor Trajectories in Fluid-Film Bearings, Adjustment of Them and Energy Efficiency Parameters
}

\author{
Leonid Savin, Sergey Mayorov, Denis Shutin and Alexander Babin \\ Oryol State University, Department of Mechatronics and International Engineering, 302020 Naugorskoe shosse 29, Oryol, Russia
}

\begin{abstract}
The problem of rotor machines modification requires development of new approaches to the design of specific nodes. As bearings are considered the most important elements of rotor machines, such approaches can imply the application of active control. In the present paper using the example of fluid-film bearings the approach is considered to enchancing energy efficiency of rotor machines by means of generating optimal trajectories. Means are shown to analyze the dynamics of a rotor system and types of the obtained trajectories. The paper also covers the approach to setting the criteria of energy efficiency of rotor's trajectories. After the optimal trajectory is obtained, active bearings are proved feasible to control the displacement of a rotor by generating a force to match the actual trajectory to the calculated one, and by this to decrease the energy loss.
\end{abstract}

\section{Introduction}

Increasing competitiveness of a machine is closely linked to the necessity of increasing their performance and decreasing size and cost at the same time. In terms of transport or technological turbomachinery, e.g. compressors, pumps, expanders, this fact requires increasing the rotational frequencies of rotors [1]. Under the conditions of high revolution frequencies and dynamic loads on the most vital parts of the machine, it seems only perspective to set the rotor on the fluid-film bearings [1-5]. Here the provision on vibrational reliability is by all means one of the main operability criteria, as it defines both the stress acting in the rotor and the dynamic load on the supporting nodes, as well as the load transferred to the housing of the machine.

\section{Nonlinear rotor dynamics problems}

The following factors play a significant role in operation of a high-speed turbomachine and influence its performance: mass and stiffness distribution, damping properties of the rotor's elements, location of the bearings, seals and dampers; type, design and operational conditions of the bearings, seals and dampers; mass and inertia characteristics of the housing of a machine; overall operational conditions; etc. It has to be noted, that the presence of the fluid-film bearings makes the dynamic analysis a complex task due to the following facts: 1) these bearings are not standard elements, and for every design and specific conditions an adequate model has to be developed; 2) can trigger self-excited, parametric and chaotic oscillations [2-4]; 3) are dependent on the combination of operational and geometric parameters in terms of their dynamic characteristics [2-6].

It is due to that, that it is reasonable to search for the rational parameters of the rotor system during the stage of project calculations. Given the present requirements the problem is almost impossible to solve without using the modern $\mathrm{CAD} / \mathrm{CAE}$ systems. In engineering practice of developing real machines there is basically no need in full modeling of the rotor system's dynamics. Instead, the following basic problems are solved [6-7]: 1) steady-state analysis; 2) determination of the natural frequencies and oscillations' shapes (modal analysis); 3) frequency response determination within the range of operational frequencies (harmonic analysis); 4) transient processes analysis, which allows modeling of the rotor's displacement caused by the action of external forces, e.g. imbalance, impulse loads (blade avulsion, various hit-like disturbances).

The motion of the rotor can be considered as of a rigid body or as of a linear elastic body. In the first approach generally one would have to solve a system of non-linear differential equations, in the second case however the task is complicated and the system now consists of differential equations in partial derivatives. Presently to solve the problems of second kind the methods of special discretization are used, e.g. finite element method, etc. [7-9]. So, when analyzing a rotor system, given the according boundary and initial conditions, the motion equations can be expressed as a system of differential equations as follows:

$$
[M] \frac{\mathrm{d}^{2}}{\mathrm{~d} t^{2}}\{Q\}+[B] \frac{\mathrm{d}}{\mathrm{d} t}\{Q\}+[K]\{Q\}=\{F\}
$$


where $\{Q\}$ - displacement vector; $[M],[B],[K]$-mass, damping and stiffness matrixes of a system 'rotor - fluidfilm bearing', which depend in a general case on displacement and time, and are defined by a number of chosen elements; $\{F\}$ - load vector.

The equation (1) can be considered as a base for all the previously mentioned types of analysis, based on which a specific task is formulated.

The boundary conditions of a model (1) are represented by external loads and reactions of the fluid film. External forces usually are known or can be statistically evaluated. Determination of the reactions of the fluid film requires the solution of the hydrodynamic equations given the cinematic parameters of the rotor [ 3 , $6,7]$. However, as it is shown in $[2-4,6,7,10]$ given the high speeds of rotors' rotation and small values of operational eccentricity, a linear representation of the fluid film reactions can be used.

The dynamic model of a rotor-bearing node given the condition of applicability of a linear approach can be represented as an oscillator on a set of springs and dampers (figure 1a).

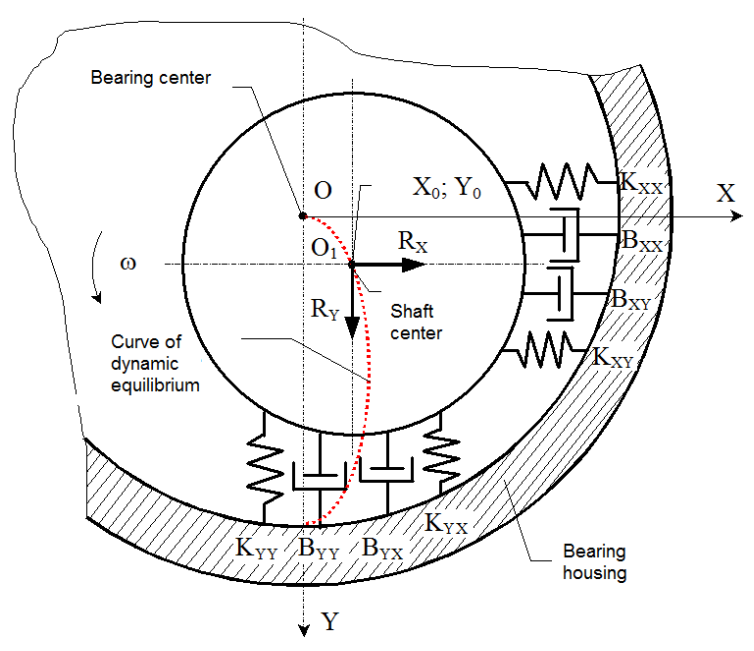

a)

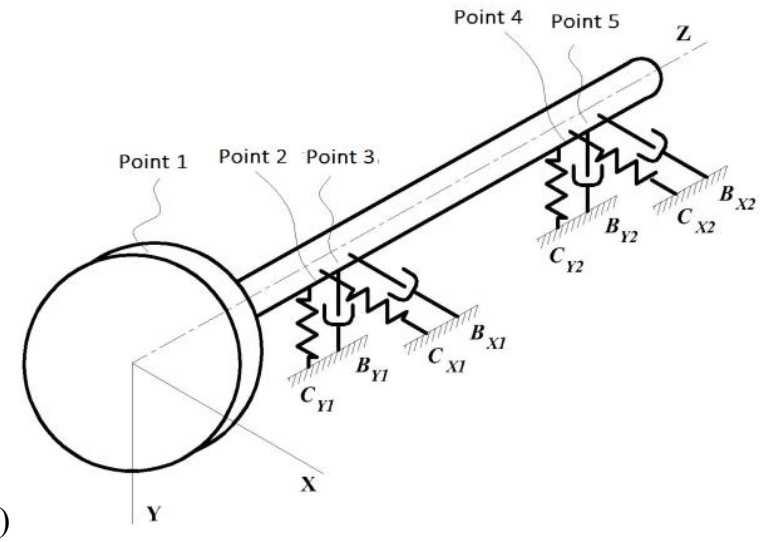

Figure 1. Dynamic model of a bearing (a) and a rotor (b)

In this case the expressions for the reactions of the fluid film will have a following form, given the small change of coordinates and velocities:

$R_{X}=R_{X 0}-K_{X X} \Delta X-K_{X Y} \Delta Y-B_{X X} \Delta \dot{X}-B_{X Y} \Delta \dot{Y}$
$R_{Y}=R_{Y 0}-K_{Y X} \Delta X-K_{Y Y} \Delta Y-B_{Y X} \Delta \dot{X}-B_{Y Y} \Delta \dot{Y}$

where the stiffness and damping coefficients are as follows:

$$
\begin{gathered}
K_{X X}=-\left(\frac{\partial R_{X}}{\partial X}\right)_{0} \quad K_{X Y}=-\left(\frac{\partial R_{X}}{\partial Y}\right)_{0} \quad K_{Y X}=-\left(\frac{\partial R_{Y}}{\partial X}\right)_{0} \\
K_{Y Y}=-\left(\frac{\partial R_{Y}}{\partial Y}\right)_{0} B_{X X}=-\left(\frac{\partial R_{X}}{\partial \dot{X}}\right)_{0} B_{X Y}=-\left(\frac{\partial R_{X}}{\partial \dot{Y}}\right)_{0} \\
B_{Y X}=-\left(\frac{\partial R_{Y}}{\partial \dot{X}}\right)_{0} B_{Y Y}=-\left(\frac{\partial R_{Y}}{\partial \dot{Y}}\right)_{0}
\end{gathered}
$$

To solve the practical application problems of dynamic analysis it is convenient to consider the reaction forces of a bearing in the following form (figure 1b):

$$
\begin{gathered}
R_{X}=R_{X 0}-C_{X} \Delta X-B_{X} \Delta \dot{X} \\
R_{Y}=R_{Y 0}-C_{Y} \Delta Y-B_{Y} \Delta \dot{Y}
\end{gathered}
$$

where the corresponding dynamic coefficients are:

$$
\begin{aligned}
& C_{X}=K_{X X}+K_{X Y} ; B_{X}=B_{X X}+B_{X Y} ; C_{Y}=K_{Y X}+K_{Y Y} \text {; } \\
& B_{Y}=B_{Y X}+B_{Y Y} .
\end{aligned}
$$

The dynamic coefficients calculation of the fluid film is an independent task. The determination of these coefficients for a specific bearing given the operational conditions in all cases but the simplest requires the development of a relatively complex models of a bearing itself, usually based on the Reynolds equation, and requires the application of numerical methods $[3,6,11]$.

Methods of numerical calculation of the dynamic coefficients for various types of bearings under the conditions of turbulent non-isothermal flow of a compressible lubricant along with some calculation results are covered in high detail in [12].

So, in order to solve practical problems, a rotor on fluid-film bearings can be considered rigid deformable body supported by a system of strings and dampers. Of particular interest here is the determination of the behavior of some character points, namely: 1 - radial load point of application; 2 and 3, 4 and 5 - points where the rotor is supported in the corresponding directions.

\section{Types of trajectories}

Trajectory method, based on the analysis of unstable processes, is an effective means of studying the influence of non-linearity of the lubricant's film's reaction and allows modeling real dynamic behavior of a rotor. The method is based on a joint numerical integration of the hydrodynamic system of equations and the equations of rotor motion. By this one can trace the influence of some specific phenomena in the fluid-film (boiling and doublephase condition) on the rotor's dynamics.

The trajectories of a rotor are the locus which determines the location of the shaft's center, the motion of which is caused by some external forces and the 
reaction forces of the fluid film, in a specific moment in time. The trajectory is a phase portrait in generalized coordinates in the shaft's center's plain. This method allows studying the dynamics of a rotor with any values of eccentricity.

The type and sizes of a trajectory of a rotor are dependent on the type and character of the load and geometric and operational parameters of the system. Generally, the values and the directions of the external forces acting on the rotor change in time.

Depending on the character of the acting forces, the following types of rotor trajectories and stability conditions of a rotor system can be highlighted:

1) point-stable (focus) (figure 2e). Such trajectory occurs when a balanced rotor moves towards the equilibrium locus curve;

2) orbit-stable (stable limit cycle), when a rotor orbits along an elliptic trajectory (figure 2d). This type of trajectories is an attribute of an unbalance rotor, which performs forced oscillations caused by the centrifugal forces; the shape of the ellipse is defined by the ratio between the inertia and imbalance forces;

3) «odd shape» - unstable-limited condition, which is characterized by an open, evolving in a limited area of the radial gap trajectory of complex shape; evidence of the presence of self-excited oscillations caused by non-linear properties of the fluid film; rotor's workability persists;

4) closed curves of complex shape, which look like cardioid (figure 2a), various epicycloids (figure $2 \mathrm{~b}$ and $2 \mathrm{c}$ ), limaçon of Pascal (figure 2d), inclined ' 8 ' (figure 2g), etc. Here rotor is exposed to precession frequent selfoscillations and overlapping them synchronous oscillations due to imbalance (bi-harmonical oscillations);

5) unstable-unlimited condition where rotor operates in an unstable region, and the trajectory is an unfolding spiral seeking the borders of the radial gap (figure $2 \mathrm{~h}$ ).

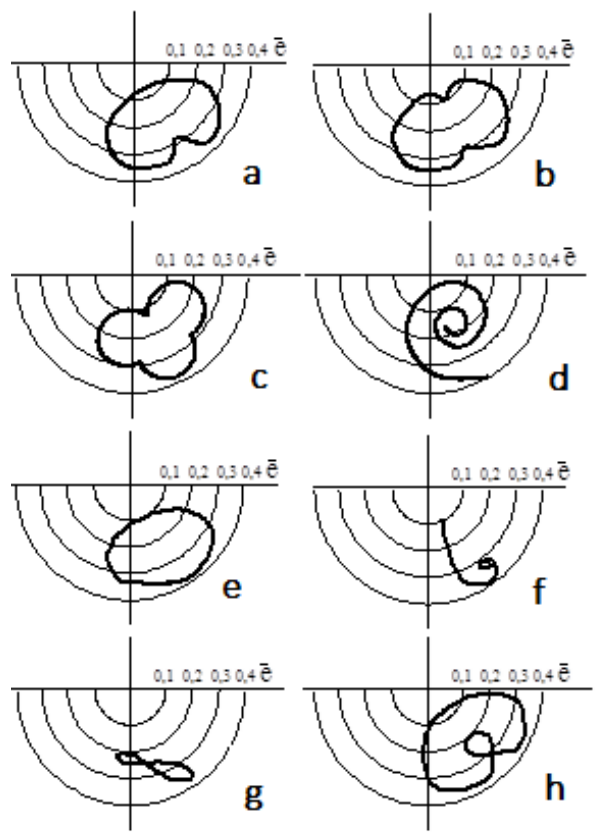

Figure 2. Trajectory types of rotors
So, the trajectory method allows evaluating the stability of the 'rotor-fluid-film bearing' system on the basis of the following assumptions:

1) the trajectory of the center of the shaft of a balanced rotor starting from some initial point ends with either a focus or a limit cycle;

2) if the motion of the rotor is unstable, the trajectory of the center of the shaft either moves monotonically from the initial position and eventually reaches the borders of the radial gap, or steadily fills some area not reaching the borders of the gap.

With that in mind, the conclusion on whether the system is stable or not is made considering the shape and the location of the trajectory.

\section{Enegry Efficiency Parameters}

As it has been previously said, the basic trend in rotor dynamics is parametric optimization, to which a big number of papers lately are dedicated. The main controversial problem of parametric optimization is the determination of the quality criteria for a specific rotor system. In the present study the criteria is suggested which is based on the effective use of the supplied energy.

The main function of any rotor system is the energy transfer from the drive to the execution device, and it is obvious that this energy characteristic can be expressed as kinetic energy of rotational motion:

$$
T_{e f f}=\frac{J \omega^{2}}{2}
$$

where $J$-inertia momentum with respect to the rotation axis; $\omega$ - rotor's angular speed.

During the revolution of the rotor in fluid-film bearings the dissipation of energy will have two distinctive components: 1) dissipation of energy due to revolution, in other words, the action of viscous forces along the angular displacement $A_{d i s}^{\text {rot }}$; 2) dissipation of energy due to oscillations, in other words, the action of the forces on trajectories considered above of the rotor $A_{d i s}^{d i s p}$; and these values can be evaluated as follows:

$$
\begin{aligned}
& A_{d i s}^{r o t}=\int_{0}^{\varphi^{*}} M_{f r} d \varphi \\
& A_{d i s}^{d i s p}=\oint_{s} \vec{R} \cdot d s
\end{aligned}
$$

where $M_{f r}$-friction momentum of a fluid-film bearing; $\vec{R}$-reaction force vector in afluid-film bearing; $d \vec{s}$ singular vector tangent to the trajectory (s) of shaft's center; $\varphi^{*}-$ angle of a rotor displacement during the time on trajectory.

So, for every closed trajectory the following energy parameter can be introduced: 


$$
E_{e f f}=\frac{A_{d i s}^{r o t}+A_{d i s}^{d i s p}}{\int_{0}^{t^{*}} T_{e f f} d t}
$$

where $t^{*}$ - time long enough to express the maximum trajectory duration.

It has to be noted, that in order to universalize the suggested criteria, it needs to be divided by the number of revolutions the rotor makes to cover the closed trajectory, so the following form is obtained:

$$
E_{\text {eff }}^{*}=\frac{\left(A_{d i s}^{r o t}+A_{d i s}^{d i s p}\right)}{\omega t^{*} \int_{0}^{t^{*}} T_{e f f} d t}
$$

The parametric optimization with a suggested criteria is indeed a laborious task in terms of calculation. One of the alternatives to it is active control of the fluid-film bearing's parameters. The variable parameters are here changed so that the trajectory with the lowest $E_{\text {eff }}^{*}$ is obtained from a number of possible.

\section{Rotor Trajectories Correction}

The problem of optimal trajectory realization in fluidfilm bearings is a natural case of a problem of trajectory determination. Such problem implies the necessity of a generated and adjustable force to act on the rotor, which would correct the rotor's location once the actual trajectory differs from the calculated. To implement this various types of modified fluid-film bearings can be used with various principles of rotor's location control. A general approach, however, remains and is in the necessity of influencing the pressure distribution in the fluid film so, that the reaction forces moved the rotor in a desired direction. The following ways of rotor's location control can be highlighted:

1) by means of adjusting the value and the configuration of the gap between a rotor and a bearing, e.g. tilting-pad bearings [13];

2) by means of adjusting the rheological properties of a lubricant in some areas of a bearing, e.g. magnetorheological lubrication [14];

3 ) by means of changing supply pressure to the area of friction, e.g. active hydrostatic bearings or hybrid bearings. In [15] a more detailed information and features of these bearings can be found. These bearings are relatively the simplest of all listed above. An active hybrid bearing is a traditional bearing with devices of supply pressure control. Modeling approach and the implementation of control in such bearings can be found in [16].

The modeling results of the described system are shown in the figure 3 . Here a rotor system was modelled on hybrid bearings and rotor was exposed to complex load - during the operation the imbalance and radial force changed.
As it is clear from the calculation results, active hybrid bearings indeed allow implementation of the rotor's location control in fluid-film bearings. In the case above application of an active hybrid bearing resulted in decreasing the amplitude of rotor's oscillations, whereas in a traditional bearing at some point, due to the centrifugal forces and a constant radial force, the fractional frequency oscillations occurred. In the active hybrid bearing the oscillations remained synchronous.
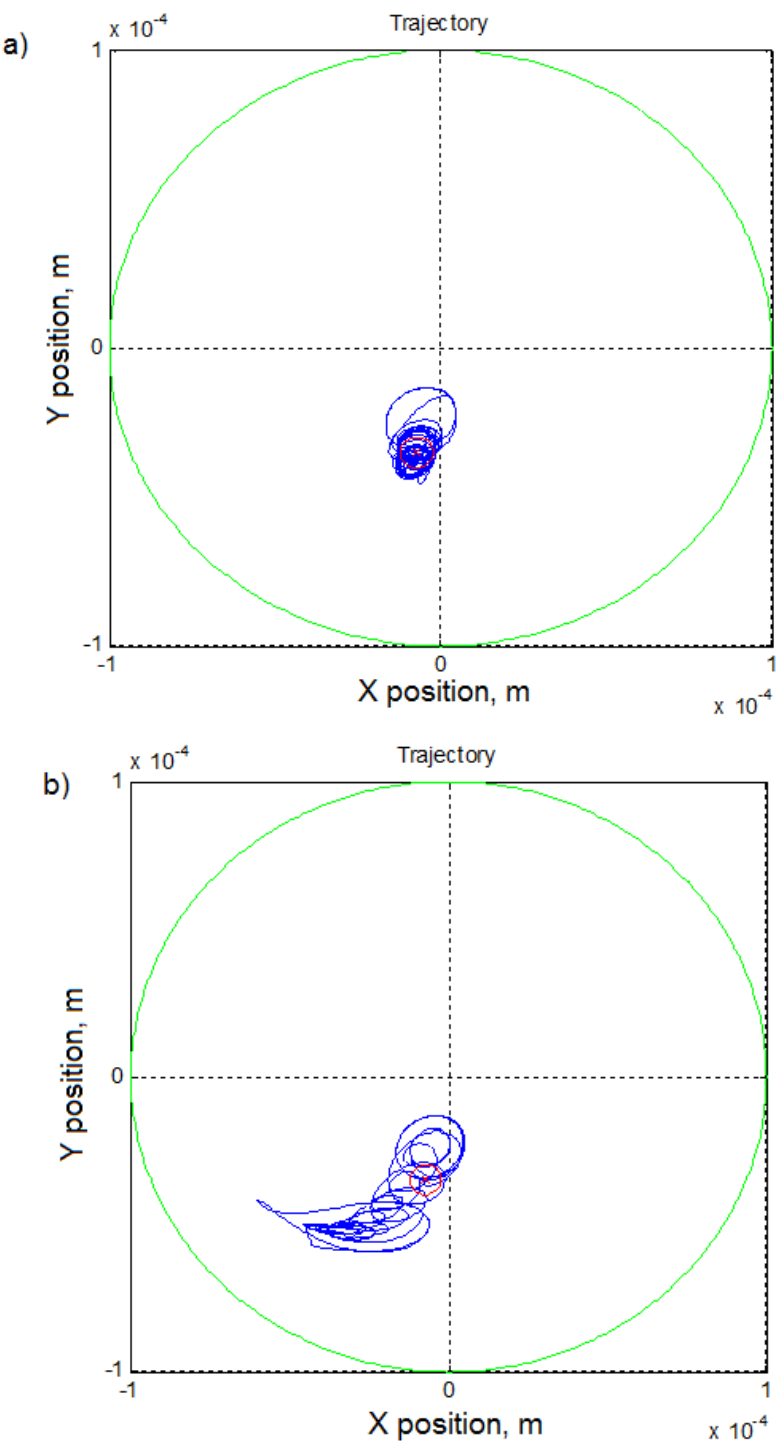

Figure 3. Trajectory types of rotors

\section{Conclusion}

The problem of rotor machines' modernization is in a close relation with development of new bearings. In this field the most perspective appear to be active fluid-film bearings. Given a relative simplicity in terms of design, they however allow actively adjusting rotor's displacement in a bearing in order to provide the most energy efficient operation. First of all, this is provided by means of lowering the friction losses, and here the determination of energy efficient trajectories is a serious calculation task. So, by combining the apparatus of optimal trajectories calculation with the principles of 
rotor's location control, it is now possible to develop bearings of new generation, which excel in terms of energy efficiency in comparison to today's solutions.

\section{Acknowledgements}

The research was implemented in the framework of the Russian Scientific Foundation project № 16-19-00186.

\section{References}

1. E. Logan, Handbook of Turbomachinery (Marcel Dekker, 1995)

2. Yu. Ravikovich, Constructions and Design of Sliding Bearings for Aircraft Engines (MAI 1995)

3. N. Arttemenko, Hydrostatic Bearings of High-Speed Rotating Machinery (Osnova 1992)

4. V. Lukanenko, Oscillations of High-Speed Rotors on Hydrostatic Bearings and Methods of Reduction of Machines Vibration Activity (Samara SC RAS 2001)

5. V. Maksimov, G. Batkis, Tribology of Fliud-Film Bearings and Seals in High-Speed Machinery. (FEN 1998)

6. M. Adams, Rotating Machinery Vibration: From Analysis to Troubleshooting (Marcel Dekker, 2001)

7. T. Yamamoto, Y. Ishida, Linear and Nonlinear Rotordynamics. A Modern Treatment with Applications (John Willey\&Sons, 2001)

8. O. Zienkiewich, R. Taylor, The Finite Element Method, 1, (Butterworth-Heinemann, 2000)

9. K. Bate, E. Wilson, Numerical Methods of Analysis and FEM (Stroyizdat, 1982)

10. O. Solomin, Dynamic Characteristics of Hybrid Bearings at the Conditions of Biphasic Lubricant
State, High School Proceedings - Mechanical Engineering, 1, 14-23 (2006)

11. A. Pugachev, M. Deckner. Shape Optimization of a Labyrinth Seal: Lleakage Minimization and Sensitivity of Rotordynamic Coefficients, IMechE Ninth International Conference on Vibrations in RotatingMachinery, 849-859

12. S.K. Guha, On the Steady-State Performance of Hydrodynamic Flexible Journal Bearings of Finite Width Lubricated By Ferro Fluids with MicroPolar Effect, International Journal of Mechanical Engineering and Robotics Research, 1, 32-49 (2012)

13. D. Deckler, R. Veillette, M. Braun, F. Choy, Simulation and Control of an Active Tilting-Pad Journal Bearing, STLE Tribology Trans, 47, 440-458 (2000)

14. J. Hesselbach, C. Abel-Keilhack, Active Hydrostatic Bearing with Magnetorheological Fluid, Journal of Applied Physics, 93, 8441-8443, (2003)

15. L. Savin, D. Shutin, Control of Rotor Motion Using Active Fluid-Film Bearings, /Proceedings of the 9th IFToMM International Conference on Rotor Dynamics. Volume 21 of the series Mechanisms and Machine Science, 1115-1122 (2015)

16. D. Shutin, R. Polyakov, L. Savin, A. Babin, Peculiarities of Reactions Control for Rotor Positioning in an Active Journal Hybrid Bearing, International Journal of Mechanics, 10, 62-67 (2003) 